\title{
Obesity and outpatient rehabilitation using mobile technologies: the potential mHealth approach
}

\author{
Gianluca Castelnuovo ${ }^{1,2}$ *, Gian Mauro Manzoni ${ }^{2,3}$, Giada Pietrabissa ${ }^{1,2}$, Stefania Corti ${ }^{2,4}$, \\ Emanuele Maria Giusti ${ }^{1}$, Enrico Molinari ${ }^{1,2}$ and Susan Simpson ${ }^{5}$ \\ 1 Department of Psychology, Catholic University of Milan, Milan, Italy \\ ${ }^{2}$ Istituto Auxologico Italiano Istituto di Ricovero e Cura a Carattere Scientifico, Psychology Research Laboratory, Ospedale San Giuseppe, Verbania, Italy \\ ${ }^{3}$ Faculty of Psychology, eCampus University, Como, Italy \\ ${ }^{4}$ Department of Psychology, University of Bergamo, Bergamo, Italy \\ ${ }^{5}$ School of Psychology, Social Work and Social Policy, University of South Australia, Adelaide, SA, Australia
}

\section{Edited by:}

Angelo Compare, University of Bergamo, Italy

\section{Reviewed by:}

Paolo Leombruni, University of Turin, Italy

Michelle Dow Keawphalouk, Harvard University, USA; MIT, USA

\section{${ }^{*}$ Correspondence:}

Gianluca Castelnuovo, Department of Psychology, Catholic University of Milan, Largo Gemelli no. 1, 20123 Milan, Italy e-mail: gianluca.castelnuovo@ unicatt.it
Obesity is currently an important public health problem of epidemic proportions (globesity). Inpatient rehabilitation interventions that aim at improving weight-loss, reducing obesity-related complications and changing dysfunctional behaviors, should ideally be carried out in a multidisciplinary context with a clinical team composed of psychologists, dieticians, psychiatrists, endocrinologists, nutritionists, physiotherapists, etc. Long-term outpatient multidisciplinary treatments are likely to constitute an essential aspect of rehabilitation. Internet-based technologies can improve long-term obesity rehabilitation within a collaborative approach by enhancing the steps specified by psychological and medical treatment protocols. These outcomes may be augmented further by the mHealth approach, through creating new treatment delivery methods to increase compliance and engagement. mHealth ( $m$-health, mobile health) can be defined as the practice of medicine and public health, supported by mobile communication devices for health services and information. mHealth applications which can be implemented in weight loss protocols and obesity rehabilitation are discussed, taking into account future research directions in this promising area.

Keywords: obesity, rehabilitation, telemedicine, telecare, mHealth, clinical psychology, health psychology, psychotherapy

\section{INTRODUCTION}

Obesity, defined as a body mass index (BMI) of $30 \mathrm{~kg} / \mathrm{m}^{2}$ or higher, is today considered an important public health problem and epidemic (globesity; Gutierrez-Fisac et al., 2006; Capodaglio and Liuzzi, 2013). Obesity is also associated with early death and universally recognized as a risk factor for many health complications and disabilities such as cardiovascular diseases, osteoarthritis, hypertension, dyslipidemia, hypercholesterolemia, Type-2 diabetes and cancer (Flegal et al., 2005; Whitlock et al., 2009; Capodaglio etal., 2010, 2011; Castelnuovo etal., 2010; Capodaglio and Liuzzi, 2013).

There is general consensus among professionals that the etiology of obesity is multifactorial with interaction between genetic, individual, and environmental factors (Marcus and Wildes, 2009). Even if genetics plays an important role in the etiology of obesity, according to Dombrowski, "Behavioral factors, i.e., poor diet and physical inactivity are among the main proximal causes linked to obesity... obesity-related morbidity... and mortality..." (p. 7, 2012). Moreover social, psychological, and psychopathological variables are clear determinants in the development and treatment of obesity (Davin and Taylor, 2009). For example, epidemiologic investigations have revealed significant correlations between obesity and eating disorders, mood disorders, anxiety disorders, and personality disorders (Hudson et al., 2007; Pickering et al., 2007; Petry et al., 2008; Scott et al., 2008; Villa et al., 2009; Manzoni et al., 2010).
In the context of in-patient rehabilitation, interventions aimed at improving weight-loss, reducing obesity-related complications and changing dysfunctional behaviors should be typically carried out in a multidisciplinary context (with a clinical team composed by, dieticians, endocrinologists or nutritionists, physiotherapists, psychiatrists, psychologists surgeons, etc.). There may be additional benefit from the inclusion of specific instructions for changing diet and self-monitoring dietary intake, whilst providing guidance and support in maintaining goals initially achieved, anticipating possible future relapses and learning strategies to cope with difficult moments or situations (Capodaglio et al., 2010, 2013a,b; Manzoni et al., 2010, 2011b; Dombrowski et al., 2011, 2012; Capodaglio and Liuzzi, 2013). A range of psychological approaches may be suitable for the in-patient treatment of obesity, such as behavioral, cognitive-behavioral, interpersonal, systemic-strategic, psychodynamic, schema etc.; Shaw etal., 2005; Castelnuovo, 2010a,b). Among these different approaches, cognitive-behavior therapy (CBT) represents the gold standard for the treatment of obesity, focusing on dysfunctional behaviors, cognitive processes, unrealistic weight goals and body image perceptions (Murphy etal., 2010). The combination of psychological therapy and diet/exercise plans, leads to better weight loss outcomes than diet/exercise interventions alone. Psychological and behavioral treatments generally include out-patient follow-up sessions which facilitate ongoing assessment an guidance in a range of areas. This may include 
determining clients' ability to self-monitor (for example, using diaries), assistance with stimulus control (for example, restricting quantities of food) and behavioral modification strategies (for example, chewing slowly, taking time to taste and enjoy food, and increasing awareness of the pleasure associated with taste; Wing, 2002; Foster et al., 2005; Swencionis and Rendell, 2012).

In a multidisciplinary obesity rehabilitation approach, it is important to underline that treatment could involve nonpharmacological, pharmacological and surgical methods. Nowadays functional anti-obesity drugs are partially indicated for those who are obese with one or more weight-related comorbid conditions (Rueda-Clausen et al., 2013; Kushner, 2014; Patham et al., 2014). Moreover additional interventions could be necessary: bariatric surgery can be an effective approach for weight loss and comorbidity reduction, taking into account that surgery can generate considerable risks and can be advised only to selected patients (Sandoval, 2011; Simpson et al., 2011; Henry et al., 2013; Kushner, 2014).

\section{OBESITY REHABILITATION NEEDS OUT-PATIENT LONG-TERM STEPS}

Recent studies have underlined the role of the neural reward system in the development and maintenance of obesity: "dysfunction of brain reward circuitry in response to food cues may predispose some individuals to obesity via an increased likelihood of overeating, particularly excessive consumption of palatable foods" (p. 744, Marcus and Wildes, 2009). Thus some kinds of obesity may be considered an expression of food "addiction," problem that typically requires a long-term treatment (Wang et al., 2001, 2002, 2004, 2009; Gearhardt et al., 2009, 2011a,b,c,d, 2012, 2013; Gearhardt and Corbin, 2011; Gearhardt and Brownell, 2013).

Moreover binge eating disorder (BED) is typically connected with obesity (American Psychiatric Association, 2000; Hill, 2005; Berkowitz and Fabricatore, 2011; Gearhardt et al., 2011c; Wilson, 2011; Schag et al., 2013; Faulconbridge and Bechtel, 2014), even if not occurring exclusively in conjunction with overweight conditions. According to Hill (2005, p. 27), "it is apparent that BED is more common in the obese than in normal-weight individuals. In US weight loss clinics, $20-40 \%$ of patients are reported to have $\mathrm{BED}$, although the use of a strict diagnostic interview reduces this to well below $20 \%$. In community samples, BED is much less common, apparent in $1-3 \%$ of respondents. Overall, the prevalence of BED in any group increases with increasing obesity." Higher levels of psychological distress and self-esteem problems are associated with obesity with BED. Typically obesity with BED requires a longer term treatment in comparison with simple obesity (Hill, 2005).

Moreover, while from a clinician's point of view a $10 \%$ weight loss is generally considered an important success due to a significant reduction in comorbidities and complications, patients typically have higher expectations, perceiving that a good result constitutes a minimal $30 \%$ body weight reduction. Thus, establishing genuine and achievable expectations of weight loss represents an important challenge for the management of obesity (Foster et al., 1997; Jeffery et al., 1998; O'Neil et al., 2000;
Wadden et al., 2000). Ongoing psychological support is required to assist patients in developing more realistic weight loss outcomes as well as in motivating them to follow rehabilitation programs (Foster et al., 2005; Nonas and Foster, 2005). Although moderate weight loss (5-10\% of initial weight) can lead to positive psychological changes, such as improvements in body satisfaction, self-esteem and mood (Hill, 2005), these findings tend to be associated with short-term studies. Typically, a long-term psychotherapeutic treatment is required in order to sustain realistic weight loss expectations and motivation to change (Hill, 2005).

Taking into account previous considerations, if we consider obesity to be a chronic form of food addiction, which may in some cases be accompanied by BED and unrealistic expectations of weight loss, long-term multidisciplinary treatment is likely to lead to optimal outcomes both across in-patient and out-patient settings.

Also, a collaborative approach, defined as a "strategy or set of strategies to help patients achieve and/or maintain a healthy weight that involve collaboration among healthcare professionals in at least two different disciplines (e.g., physicians and dieticians) for the delivery of weight management interventions" (p. 1190, Rao etal., 2011) is required. Thus strategies based on central planning, grounded in a "chronic care model" logic, tend to obtain better results, although at this early stage only a limited number of articles have reported real and practical collaborative experiences, largely in out-patient settings (Rao et al., 2011).

\section{NEW TECHNOLOGIES FOR OUT-PATIENT OBESITY REHABILITATION: THE TECNOB PROJECT}

Internet-based technologies provide patients with continuous and remote psychological, medical and nutritional support and education in order to enhance motivation, compliance and engagement, thereby maximizing the benefits of collaborative outpatient rehabilitation programs (Castelnuovo et al., 2003, 2010, 2011a,b,c,d; Riva et al., 2006; Castelnuovo and Simpson, 2011; Manzoni et al., 2011a; Rao et al., 2011; Simpson and Slowey, 2011).

Moreover the use of telemonitoring and telecare approaches that ensure continuity of care in out-patient settings can contribute to a significant cost reduction in the management of obesity and other chronic pathologies (Ekeland et al., 2010,2011; Manzoni et al., 2011a).

One such pioneering example of a collaborative approach is the TECNOB Project (TEChNology for OBesity; Castelnuovo et al., 2003, 2010, 2011a,b; Castelnuovo, 2007). It runs for a total duration of 13 months and consists of two consecutive phases: in-patient ( 1 month) and out-patient (the following 12 months). The clinician-patient relationship is considered a highly significant agent and vehicle for change. After discharge, out-patients begin to experience a sense of autonomy and competence as they continue the change process they have begun to develop during the in-patient phase, whilst learning to face a range of resistances and barriers. Through the use of videoconferencing out-patients are supported by the clinicians who worked with them during the in-hospital phase, through exploring resistances and impediments they experience and finding functional and healthy coping 
mechanisms. Furthermore, out-patients are helped to experience a sense of mastery as they become proficient at attaining healthy behavioral changes.

Other positive experiences are well reported and described in (Bacigalupo et al., 2013), where the common components of the Internet-based clinical protocols are few (self-monitoring related to weight and physical activity and automatic-professional feedback to participants), whereas the intervention programs varied significantly in many details and features (Bacigalupo et al., 2013).

\section{NEW TECHNOLOGIES FOR OUT-PATIENT OBESITY REHABILITATION: THE mHealth SCENARIO}

Internet-based tools can provide promising results in enhancing weight reduction among obese patients but further studies are required in order to determine its long-term efficacy and effectiveness across clinical, organizational, and economic perspectives. (Manzoni etal., 2008, 2011a; Khaylis et al., 2010; Rao et al., 2011).

Until now the published data has not supported the competitive use of Internet interventions for weight loss and maintenance in out-patient settings. In spite of this lack of literature, promising clinical reports have been published about the usefulness of mobile phone devices in promoting healthy habits and weight loss attitudes (Rao et al., 2011; Park and Kim, 2012; Pellegrini etal., 2012; Schiel et al., 2012; Bacigalupo et al., 2013; Hebden et al., 2013; Rodrigues et al., 2013; Schoffman et al., 2013; Sharifi et al., 2013; Shaw et al., 2013).

Moreover, no unequivocal data have been collected about real costs of telemedicine. Certainly it could reduce travel time, hospital admissions and indirect costs to service users and their social networks (Ekeland et al., 2010, 2011; Khaylis et al., 2010; Hilty et al., 2013). The potential for technical problems as well as skepticism or reticence from patients, caregivers, nurses, and physicians may limit the spread of e-health solutions (e.g., Rees and Stone, 2005). The mHealth approach has the potential to make contributions not only in adult obesity (Tufano and Karras, 2005; Burke et al., 2012) but also in pediatric obesity (Jensen et al., 2012; Turner-McGrievy etal., 2013), thereby creating new treatment delivery methods that could increase participation, compliance and engagement (Graffigna et al., 2013a,b). About pediatric obesity, Cohen et al. (2012) in a recent review noted that telemedicine could be a promising approach to pediatric weight management, particularly for families in rural contexts with limited access to traditional treatments, even if many doubts are present again, particularly what treatment components (psychological support, lifestyle modification, nutritional education, medical prescription, etc.) could functionally fit into the e-health settings.

According to Eysenbach (2001, p. 1), e-health could be defined as "an emerging field in the intersection of medical informatics, public health and business, referring to health services and information delivered or enhanced through the Internet and related technologies. In a broader sense, the term characterizes not only a technical development, but also a state-of-mind, a way of thinking, an attitude, and a commitment for networked, global thinking, to improve health care locally, regionally, and worldwide by using information and communication technology." e-health is characterized by the presence of 10 features: efficiency, enhanced quality of care, evidence-based approach, empowerment of consumers and patients, encouragement of a new relationship between the patient and health professional, on-line education of physicians, information and communication exchange, extension of the health care scope beyond its conventional boundaries (in both geographical and conceptual sense), ethics and equity (Eysenbach, 2001). mHealth ( $\mathrm{m}$-health, mhealth, mobile health) could be defined as the practice of medicine and public health, supported by mobile communication devices, such as mobile phones, tablet computers, and PDAs, for health services and information (Riper et al., 2010; Eysenbach, 2011; Cipresso et al., 2012; Whittaker, 2012; Fiordelli etal., 2013). mHealth applications have also been implemented with promising applications and results in weight loss protocols and obesity rehabilitation (Chomutare etal., 2011; Burke etal., 2012; Cafazzo etal., 2012; Fiordelli et al., 2013; Martinez-Perez et al., 2013; Turner-McGrievy et al., 2013).

\section{FIVE PSYCHOLOGICAL COMPONENTS TO BE CONSIDERED IN mHealth WEIGHT-LOSS APPLICATIONS}

According to Khaylis et al. (2010, p. 932-936) five psychological components need to be considered for technology-based and mHealth-based obesity rehabilitation in order to facilitate weight-loss.

\section{SELF-MONITORING}

Self-monitoring refers to the process in which individuals regulate and keep track of their own behaviors. Technology can simplify the monitoring process, recording one's progress of food intake and physical activity using online devices. The reason these technologies are likely to be effective is because portable body monitors, pedometers, and handheld PDAs are mobile and, therefore, can be easily used, resulting in continuous self-monitoring. Also, these devices are more convenient for individuals without access to a high-speed Internet connection.

\section{COUNSELOR FEEDBACK AND COMMUNICATION}

Feedback from a counselor regarding goals, progress, and results can encourage, motivate, and assist patients in successfully completing a weight-loss program. A functional approach is to provide online weight-loss interventions with brief weekly or monthly counselor or psychologist visits. Participants typically submit their weekly food and exercise journals online, receiving personalized feedback, reinforcement, and recommendations from a counselor over e-mail.

\section{SOCIAL SUPPORT}

A group treatment format is typically preferred for behavioral weight-loss interventions. Not only does it constitute a costeffective method for delivering treatment to a larger number of people, but it also enhances social support, an important facilitator of behavioral change. Group support can foster motivation, encouragement, and commonality. To facilitate communication among participants electronic message boards, forums, "real time" chat rooms or online meetings represent useful tools. 


\section{STRUCTURED PROGRAM}

Technology-based weight-loss programs incorporate principles of behavior therapy and change. They consist of structured weekly lessons on various topics, including nutrition, exercise, stimulus control, self-regulation strategies, goal-setting.

\section{INDIVIDUALLY TAILORED PROGRAM}

Interventions specifically designed around individual's goals typically record higher rates of adherence and weight loss. In one report, participants were required to meet with a health coach and select four high-priority behavioral change goals, before being monitored through a behavioral skills training program. In another study real-time SMS text messages were delivered to each patient, as a direct challenge to pre-identified barriers to exercise.

\section{FUTURE DIRECTIONS IN CLINICAL PSYCHOLOGY FOR OBESITY REHABILITATION}

Potential benefits of mobile monitoring methods for behavioral weight loss protocols appear clear (Turner-McGrievy et al., 2013). However, "future studies should examine ways to predict which self-monitoring method works best for an individual to increase adherence" (Turner-McGrievy et al., 2013, p. 513). There is a critical need for scientific research to evaluate the specific outcomes of collaborative approaches for weight management that utilize Internet and mobile based tools.

mHealth approach could help clinicians by motivating patients in remote settings to develop healthier lifestyles (Castelnuovo, 2010a; Pietrabissa et al., 2012), to accept more intrusive medical treatments (such as drugs and weight-loss surgery), to cope with chronic conditions and to reduce complications (such as Type-2 Diabetes, hypertension and cardiovascular disease; Nguyen and Lau, 2012).

Moreover clinicians should adhere to good professional practice protocols in technological settings: "discussions of weight should be performed in a non-judgmental, respectful, and unhurried manner" (p. 1200, Rao et al., 2011), "readiness and self-efficacy to change behaviors should be assessed before weight loss strategies are initiated, and this information should be factored into decisions about what type of approach to use" (p. 1200, 2011), and collaborative approaches involving physicians, psychologists, nurses, and other clinicians need to be considered by utilizing consistent planning and training modalities.

Future directions in obesity and weight-reduction research have been provided by Rao et al. (2011, p. 1200):

(1) "There is a need for larger studies, both those that include technologically based interventions and those that do not, that enroll a diverse spectrum of overweight and obese patients in terms of sex, race, and socioeconomic status. Latino subjects and men, in particular, are underrepresented in obesity studies to date. There is also a need to investigate the specific features of technologically based interventions (e.g., content, format, device) that make such interventions successful in promoting weight loss.

(2) Because attrition rates from technology-based studies are very high, there is a need to develop effective strategies to keep patients engaged in using technology tools for the long-term.
(3) Further evaluation of collaborative approaches (e.g., approaches involving centralized planning, approaches involving nurses in intervention delivery) in general is needed. In particular, larger studies of longer duration are needed to evaluate the effectiveness of the chronic care model as a framework for weight management interventions.

(4) Use of electronic health records is increasing, and there is a need to explore the use of these valuable tools, not only for identification and assessment of obesity but also for the delivery of obesity interventions."

Whittaker (2012, p. 6) provides some methodological suggestions for future research in this issue: "evaluations of effectiveness and usability are required and should be made publicly available. Where evaluation is planned during the development stage, data collection can be built in as an integral part of the program. The ideal of randomized controlled trials will still be necessary in some contexts. In these cases, careful consideration should be given to the appropriate comparator to ensure the right question is being answered. For example, what is usual care for this target audience? Can we measure an improvement in access as an outcome? Other research methods will be more appropriate in other circumstances, such as adaptive trials to allow the intervention to develop and improve as part of the research; observational trials and qualitative research methods to detect unintended consequences and changes to workflow; and qualitative studies to test acceptability. Evaluating effectiveness and usability is also possible while implementing a system, for example, with novel designs such as the stepped wedge cluster randomized trial, and particularly where there is little likelihood of harm."

To conclude, further studies should investigate both possible advantages and applications of Internet and mHealth technologies in the treatment of obesity. In spite of promising preliminary reports, the evidence-base for the effectiveness of mHealth applications is meager and it remains too early to be able to recommend it for use in clinical settings.

\section{REFERENCES}

American Psychiatric Association. (2000). Diagnostic and Statistical Manual of Mental Disorders, 4th Edn (Washington, DC: American Psychiatric Association).

Bacigalupo, R., Cudd, P., Littlewood, C., Bissell, P., Hawley, M. S., and Buckley Woods, H. (2013). Interventions employing mobile technology for overweight and obesity: an early systematic review of randomized controlled trials. Obes. Rev. 14, 279-291. doi: 10.1111/obr.12006

Berkowitz, R. I., and Fabricatore, A. N. (2011). Obesity, psychiatric status, and psychiatric medications. Psychiatr. Clin. North Am. 34, 747-764. doi: 10.1016/j.psc.2011.08.007

Burke, L. E., Styn, M. A., Sereika, S. M., Conroy, M. B., Ye, L., Glanz, K., et al. (2012). Using mHealth technology to enhance self-monitoring for weight loss: a randomized trial. Am. J. Prev. Med. 43, 20-26. doi: 10.1016/j.amepre.2012.03.016

Cafazzo, J. A., Casselman, M., Hamming, N., Katzman, D. K., and Palmert, M. R. (2012). Design of an mHealth app for the self-management of adolescent type 1 diabetes: a pilot study. J. Med. Internet Res. 14:e70. doi: 10.2196/jmir.2058

Capodaglio, P., Capodaglio, E. M., Precilios, H., Vismara, L., Tacchini, E., Finozzi, E., et al. (2011). Obesity and work: an emerging problem. G. Ital. Med. Lav. Ergon. $33,47-54$.

Capodaglio, P., Castelnuovo, G., Brunani, A., Vismara, L., Villa, V., and Capodaglio, E. M. (2010). Functional limitations and occupational issues in obesity: a review. Int. J. Occup. Saf. Ergon. 16, 507-523. 
Capodaglio, P., Cimolin, V., Tacchini, E., Precilios, H., and Brunani, A. (2013a). Effectiveness of in-patient rehabilitation in obesity-related orthopedic conditions. J. Endocrinol. Invest. 36, 628-631. doi: 10.3275/8897

Capodaglio, P., Lafortuna, C., Petroni, M. L., Salvadori, A., Gondoni, L., Castelnuovo, G., et al. (2013b). Rationale for hospital-based rehabilitation in obesity with comorbidities. Eur. J. Phys. Rehabil. Med. 49, 399-417.

Capodaglio, P., and Liuzzi, A. (2013). Obesity: a disabling disease or a condition favoring disability? Eur. J. Phys. Rehabil. Med. 49, 395-398.

Castelnuovo, G. (2007). Move the healthcare where it really needs: TECNOB project to improve outpatient obesity treatment. (E-letter in response to Wister, Loewen, Kennedy-Symonds, McGowan, McCoy, Singer, "One-year follow-up of a therapeutic lifestyle intervention targeting cardiovascular disease risk.”) CMAJ 177, 859-865. doi: 10.1503/cmaj.061059

Castelnuovo, G. (2010a). Empirically supported treatments in psychotherapy: towards an evidence-based or evidence-biased psychology in clinical settings? Front. Psychol. 1:27. doi: 10.3389/fpsyg.2010.00027

Castelnuovo, G. (2010b). No medicine without psychology: the key role of psychological contribution in clinical settings. Front. Psychol. 1:4. doi: 10.3389/fpsyg.2010.00004

Castelnuovo, G., Gaggioli, A., Mantovani, F., and Riva, G. (2003). From psychotherapy to e-therapy: the integration of traditional techniques and new communication tools in clinical settings. Cyberpsychol. Behav. 6, 375-382. doi: 10.1089/109493103322278754

Castelnuovo, G., Manzoni, G. M., Corti, S., Cuzziol, P., Villa, V., and Molinari, E. (2011a). "Clinical psychology and medicine for the treatment of obesity in out-patient settings: the TECNOB project," in Telemedicine Techniques and Applications, eds G. Graschew and S. Rakowsky (Rijeka: InTech), 257-268.

Castelnuovo, G., Manzoni, G. M., Cuzziol, P., Cesa, G. L., Corti, S., Tuzzi, C., et al. (2011b). TECNOB study: ad interim results of a randomized controlled trial of a multidisciplinary telecare intervention for obese patients with type-2 diabetes. Clin. Pract. Epidemiol. Ment. Health 7, 44-50. doi: 10.2174/1745017901107010044

Castelnuovo, G., Manzoni, G. M., Villa, V., Cesa, G. L., and Molinari, E. (2011c). Brief strategic therapy vs cognitive behavioral therapy for the inpatient and telephonebased outpatient treatment of binge eating disorder: the STRATOB randomized controlled clinical trial. Clin. Pract. Epidemiol. Ment. Health 7, 29-37. doi: 10.2174/1745017901107010029

Castelnuovo, G., Manzoni, G. M., Villa, V., Cesa, G. L., Pietrabissa, G., and Molinari, E. (2011d). The STRATOB study: design of a randomized controlled clinical trial of Cognitive Behavioral Therapy and Brief Strategic Therapy with telecare in patients with obesity and binge-eating disorder referred to residential nutritional rehabilitation. Trials 12:114. doi: 10.1186/1745-6215-12-114

Castelnuovo, G., Manzoni, G. M., Cuzziol, P., Cesa, G. L., Tuzzi, C., Villa, V., et al. (2010). TECNOB: study design of a randomized controlled trial of a multidisciplinary telecare intervention for obese patients with type-2 diabetes. BMC Public Health 10:204. doi: 10.1186/1471-2458-10-204

Castelnuovo, G., and Simpson, S. (2011). Ebesity - e-health for obesity - new technologies for the treatment of obesity in clinical psychology and medicine. Clin. Pract. Epidemiol. Ment. Health 7, 5-8. doi: 10.2174/17450179011070 10005

Chomutare, T., Fernandez-Luque, L., Arsand, E., and Hartvigsen, G. (2011). Features of mobile diabetes applications: review of the literature and analysis of current applications compared against evidence-based guidelines. J. Med. Internet Res. 13:e65. doi: 10.2196/jmir.1874

Cipresso, P., Serino, S., Villani, D., Repetto, C., Selitti, L., Albani, G., et al. (2012). Is your phone so smart to affect your states? An exploratory study based on psychophysiological measures. Neurocomputing 84, 23-30. doi: 10.1016/j.neucom.2011.12.027

Cohen, G. M., Irby, M. B., Boles, K., Jordan, C., and Skelton, J. A. (2012). Telemedicine and Pediatric Obesity Treatment: review of the literature and lessons learned. Clin. Obes. 2, 103-111. doi: 10.1111/j.1758-8111.2012. 00050.x

Davin, S. A., and Taylor, N. M. (2009). Comprehensive review of obesity and psychological considerations for treatment. Psychol. Health Med. 14, 716-725. doi: 10.1080/13548500903431501

Dombrowski, S. U., Avenell, A., and Sniehott, F. F. (2011). Behavioral interventions for obese adults with additional risk factors for morbidity: systematic review of effects on behavior, weight and disease risk factors. Obes. Facts 3, 377-396. doi: $10.1159 / 000323076$

Dombrowski, S. U., Sniehotta, F. F., Avenell, A., Johnston, M., MacLennan, G., and Araújo-Soares, V. (2012). Identifying active ingredients in complex behavioral interventions for obese adults with obesity-related co-morbidities or additional risk factors for co-morbidities: a systematic review. Health Psychol. Rev. 6, 7-32. doi: 10.1080/17437199.2010.513298

Ekeland, A. G., Bowes, A., and Flottorp, S. (2010). Effectiveness of telemedicine: a systematic review of reviews. Int. J. Med. Inform. 79, 736-771. doi: 10.1016/j.ijmedinf.2010.08.006

Ekeland, A. G., Bowes, A., and Flottorp, S. (2011). Methodologies for assessing telemedicine: a systematic review of reviews. Int. J. Med. Inform. 81, 1-11. doi: 10.1016/j.ijmedinf.2011.10.009

Eysenbach, G. (2001). What is e-health? J. Med. Internet Res. 3:e20. doi: 10.2196/jmir.3.2.e20

Eysenbach, G. (2011). Can tweets predict citations? Metrics of social impact based on Twitter and correlation with traditional metrics of scientific impact. J. Med. Internet Res. 13:e123. doi: 10.2196/jmir.2012

Faulconbridge, L. F., and Bechtel, C. F. (2014). Depression and Disordered Eating in the Obese Person. Curr. Obes. Rep. 3, 127-136. doi: 10.1007/s13679-0130080-9

Fiordelli, M., Diviani, N., and Schulz, P. J. (2013). Mapping mHealth research: a decade of evolution. J. Med. Internet Res. 15, e95. doi: 10.2196/jmir.2430

Flegal, K. M., Graubard, B. I., Williamson, D. F., and Gail, M. H. (2005). Excess deaths associated with underweight, overweight, and obesity. JAMA 293, 1861-1867. doi: 10.1001/jama.293.15.1861

Foster, G. D., Makris, A. P., and Bailer, B. A. (2005). Behavioral treatment of obesity. Am. J. Clin. Nutr. 82(Suppl. 1), 230S-235S.

Foster, G. D., Wadden, T. A., Vogt, R. A., and Brewer, G. (1997). What is a reasonable weight loss? Patients' expectations and evaluations of obesity treatment outcomes. J. Consult. Clin. Psychol. 65, 79-85. doi: 10.1037/0022-006X. 65.1 .79

Gearhardt, A., Roberts, M., and Ashe, M. (2013). If sugar is addictive...what does it mean for the law? J. Law Med. Ethics 41(Suppl. 1), 46-49. doi: 10.1111/jlme. 12038

Gearhardt, A. N., and Brownell, K. D. (2013). Can food and addiction change the game? Biol. Psychiatry 73, 802-803. doi: 10.1016/j.biopsych.2012.07.024

Gearhardt, A. N., and Corbin, W. R. (2011). The role of food addiction in clinical research. Curr. Pharm. Des. 17, 1140-1142. doi: 10.2174/1381612117956 56800

Gearhardt, A. N., Corbin, W. R., and Brownell, K. D. (2009). Food addiction: an examination of the diagnostic criteria for dependence. J. Addict. Med. 3, 1-7. doi: 10.1097/ADM.0b013e318193c993

Gearhardt, A. N., Davis, C., Kuschner, R., and Brownell, K. D. (2011a). The addiction potential of hyperpalatable foods. Curr. Drug Abuse Rev. 4, 140-145. doi: $10.2174 / 1874473711104030140$

Gearhardt, A. N., Grilo, C. M., DiLeone, R. J., Brownell, K. D., and Potenza, M. N. (2011b). Can food be addictive? Public health and policy implications. Addiction 106, 1208-1212. doi: 10.1111/j.1360-0443.2010.03301.x

Gearhardt, A. N., White, M. A., and Potenza, M. N. (2011c). Binge eating disorder and food addiction. Curr. Drug Abuse Rev. 4, 201-207. doi: $10.2174 / 1874473711104030201$

Gearhardt, A. N., Yokum, S., Orr, P. T., Stice, E., Corbin, W. R., and Brownell, K. D. (2011d). Neural correlates of food addiction. Arch. Gen. Psychiatry 68, 808-816. doi: 10.1001/archgenpsychiatry.2011.32

Gearhardt, A. N., White, M. A., Masheb, R. M., Morgan, P. T., Crosby, R. D., and Grilo, C. M. (2012). An examination of the food addiction construct in obese patients with binge eating disorder. Int. J. Eat. Disord. 45, 657-663. doi: 10.1002/eat.20957

Graffigna, G., Barello, S., and Riva, G. (2013a). Technologies for patient engagement. Health Aff. (Millwood) 32, 1172. doi: 10.1377/hlthaff.20 13.0279

Graffigna, G., Barello, S., Wiederhold, B. K., Bosio, A. C., and Riva, G. (2013b). Positive technology as a driver for health engagement. Stud. Health Technol. Inform. 191, 9-17. doi: 10.3233/978-1-61499-282-0-9

Gutierrez-Fisac, J. L., Angel Royo-Bordonada, M., and Rodriguez-Artalejo, F. (2006). Health-risks associated with Western diet and sedentariness: the obesity epidemia. Gac. Sanit. 20(Suppl. 1), 48-54. doi: 10.1157/13086026 
Hebden, L., Balestracci, K., McGeechan, K., Denney-Wilson, E., Harris, M., Bauman, A., et al. (2013). 'TXT2BFiT' a mobile phone-based healthy lifestyle program for preventing unhealthy weight gain in young adults: study protocol for a randomized controlled trial. Trials 14:75. doi: 10.1186/1745-621514-75

Henry, R. R., Chilton, R., and Garvey, W. T. (2013). New options for the treatment of obesity and type 2 diabetes mellitus (narrative review). J. Diabetes Complications 27, 508-518. doi: 10.1016/j.jdiacomp.2013.04.011

Hill, A. J. (2005). Psychological aspects of obesity. Psychiatry 4, 26-30. doi: 10.1383/psyt.4.4.26.63443

Hilty, D. M., Ferrer, D. C., Parish, M. B., Johnston, B., Callahan, E. J., and Yellowlees, P. M. (2013). The effectiveness of telemental health: a 2013 review. Telemed. J. E Health 19, 444-454. doi: 10.1089/tmj.2013.0075

Hudson, J. I., Hiripi, E., Pope, H. G. Jr., and Kessler, R. C. (2007). The prevalence and correlates of eating disorders in the National Comorbidity Survey Replication. Biol. Psychiatry 61, 348-358. doi: 10.1016/j.biopsych.2006.03.040

Jeffery, R. W., Wing, R. R., and Mayer, R. R. (1998). Are smaller weight losses or more achievable weight loss goals better in the long term for obese patients? J. Consult Clin. Psychol. 66, 641-645. doi: 10.1037/0022-006X.66. 4.641

Jensen, C. D., Aylward, B. S., and Steele, R. G. (2012). Predictors of attendance in a practical clinical trial of two pediatric weight management interventions. Obesity (Silver Spring) 20, 2250-2256. doi: 10.1038/oby.2012.96

Khaylis, A., Yiaslas, T., Bergstrom, J., and Gore-Felton, C. (2010). A review of efficacious technology-based weight-loss interventions: five key components. Telemed. J. E Health 16, 931-938. doi: 10.1089/tmj.2010.0065

Kushner, R. F. (2014). Weight loss strategies for treatment of obesity. Prog. Cardiovasc. Dis. 56, 465-472. doi: 10.1016/j.pcad.2013.09.005

Manzoni, G. M., Castelnuovo, G., and Molinari, E. (2008). Weight loss with a lowcarbohydrate, Mediterranean, or low-fat diet. N. Engl. J. Med. 359, 229-241. doi: 10.1056/NEJMoa0708681

Manzoni, G. M., Cribbie, R. A., Villa, V., Arpin-Cribbie, C. A., Gondoni, L., and Castelnuovo, G. (2010). Psychological well-being in obese inpatients with ischemic heart disease at entry and at discharge from a four-week cardiac rehabilitation program. Front. Psychol. 1:38. doi: 10.3389/fpsyg.2010.00038

Manzoni, G. M., Pagnini, F., Corti, S., Molinari, E., and Castelnuovo, G. (2011a). Internet-based behavioral interventions for obesity: an updated systematic review. Clin. Pract. Epidemiol. Ment. Health 7, 19-28. doi: 10.2174/1745017901107010019

Manzoni, G. M., Villa, V., Compare, A., Castelnuovo, G., Nibbio, F., Titon, A. M., et al. (2011b). Short-term effects of a multi-disciplinary cardiac rehabilitation programme on psychological well-being, exercise capacity and weight in a sample of obese in-patients with coronary heart disease: a practice-level study. Psychol. Health Med. 16, 178-189. doi: 10.1080/13548506.2010.542167

Marcus, M. D., and Wildes, J. E. (2009). Obesity: is it a mental disorder? Int. J. Eat. Disord. 42, 739-753. doi: 10.1002/eat.20725

Martinez-Perez, B., de la Torre-Diez, I., and Lopez-Coronado, M. (2013). Mobile health applications for the most prevalent conditions by the World Health Organization: review and analysis. J. Med. Internet Res. 15:e120. doi: 10.2196/jmir.2600

Murphy, R., Straebler, S., Cooper, Z., and Fairburn, C. G. (2010). Cognitive behavioral therapy for eating disorders. Psychiatr. Clin. North Am. 33, 611-627. doi: 10.1016/j.psc.2010.04.004

Nguyen, T., and Lau, D. C. (2012). The obesity epidemic and its impact on hypertension. Can. J. Cardiol. 28, 326-333. doi: 10.1016/j.cjca.2012.01.001

Nonas, C. A., and Foster, G. D. (2005). Setting achievable goals for weight loss. J. Am. Diet. Assoc. 105(Suppl. 1), S118-S123. doi: 10.1016/j.jada.2005. 02.030

O'Neil, P. M., Smith, C. F., Foster, G. D., and Anderson, D. A. (2000). The perceived relative worth of reaching and maintaining goal weight. Int. J. Obes. Relat. Metab. Disord. 24, 1069-1076. doi: 10.1038/sj.ijo.0801242

Park, M. J., and Kim, H. S. (2012). Evaluation of mobile phone and Internet intervention on waist circumference and blood pressure in post-menopausal women with abdominal obesity. Int. J. Med. Inform. 81, 388-394. doi: 10.1016/j.ijmedinf.2011.12.011

Patham, B., Mukherjee, D., and San Juan, Z. T. (2014). Contemporary review of drugs used to treat obesity. Cardiovasc. Hematol. Agents Med. Chem. 11, 272-280. doi: $10.2174 / 1871525712666140317162655$
Pellegrini, C. A., Duncan, J. M., Moller, A. C., Buscemi, J., Sularz, A., DeMott, A., et al. (2012). A smartphone-supported weight loss program: design of the ENGAGED randomized controlled trial. BMC Public Health 12:1041. doi: 10.1186/14712458-12-1041

Petry, N. M., Barry, D., Pietrzak, R. H., and Wagner, J. A. (2008). Overweight and obesity are associated with psychiatric disorders: results from the National Epidemiologic Survey on Alcohol and Related Conditions. Psychosom. Med. 70, 288-297. doi: 10.1097/PSY.0b013e3181651651

Pickering, R. P., Grant, B. F., Chou, S. P., and Compton, W. M. (2007). Are overweight, obesity, and extreme obesity associated with psychopathology? Results from the national epidemiologic survey on alcohol and related conditions. J. Clin. Psychiatry 68, 998-1009. doi: 10.4088/JCP.v68 n0704

Pietrabissa, G., Manzoni, G. M., Corti, S., Vegliante, N., Molinari, E., and Castelnuovo, G. (2012). Addressing motivation in globesity treatment: a new challenge for clinical psychology. Front. Psychol. 3:317. doi: 10.3389/fpsyg.2012. 00317

Rao, G., Burke, L. E., Spring, B. J., Ewing, L. J., Turk, M., Lichtenstein, A. H., et al. (2011). New and emerging weight management strategies for busy ambulatory settings: a scientific statement from the American Heart Association endorsed by the Society of Behavioral Medicine. Circulation 124, 1182-1203. doi: 10.1161/CIR.0b013e31822b9543

Rees, C. S., and Stone, S. (2005). Therapeutic alliance in face- to-face versus videoconferenced psychotherapy. Prof. Psychol. Res. Pr. 36, 649-653. doi: 10.1037/0735-7028.36.6.649

Riper, H., Andersson, G., Christensen, H., Cuijpers, P., Lange, A., and Eysenbach, G. (2010). Theme issue on e-mental health: a growing field in internet research. J. Med. Internet Res. 12:e74. doi: 10.2196/jmir.1713

Riva, G., Castelnuovo, G., and Mantovani, F. (2006). Transformation of flow in rehabilitation: the role of advanced communication technologies. Behav. Res. Methods 38, 237-244. doi: 10.3758/BF03192775

Rodrigues, J. J., Lopes, I. M., Silva, B. M., and Torre Ide, L. (2013). A new mobile ubiquitous computing application to control obesity: SapoFit. Inform. Health Soc. Care 38, 37-53. doi: 10.3109/17538157.2012.674586

Rojas, S. V., and Gagnon, M. P. (2008). A systematic review of the key indicators for assessing telehomecare cost-effectiveness. Telemed. J. E Health 14, 896-904. doi: 10.1089/tmj.2008.0009

Rueda-Clausen, C. F., Padwal, R. S., and Sharma, A. M. (2013). New pharmacological approaches for obesity management. Nat. Rev. Endocrinol. 9, 467-478. doi: 10.1038/nrendo.2013.113

Sandoval, D. (2011). Bariatric surgeries: beyond restriction and malabsorption. Int. J. Obes. (Lond.) 35(Suppl. 3), S45-S49. doi: 10.1038/ijo.2011.148

Schag, K., Schonleber, J., Teufel, M., Zipfel, S., and Giel, K. E. (2013). Food-related impulsivity in obesity and binge eating disorder-a systematic review. Obes. Rev. 14, 477-495. doi: 10.1111/obr.12017

Schiel, R., Kaps, A., and Bieber, G. (2012). Electronic health technology for the assessment of physical activity and eating habits in children and adolescents with overweight and obesity IDA. Appetite 58, 432-437. doi: 10.1016/j.appet.2011.11.021

Schoffman, D. E., Turner-McGrievy, G., Jones, S. J., and Wilcox, S. (2013). Mobile apps for pediatric obesity prevention and treatment, healthy eating, and physical activity promotion: just fun and games? Trans. Behav. Med. 3, 320-325. doi: 10.1007/s13142-013-0206-3

Scott, K. M., Bruffaerts, R., Simon, G. E., Alonso, J., Angermeyer, M., de Girolamo, G., et al. (2008). Obesity and mental disorders in the general population: results from the world mental health surveys. Int. J. Obes. (Lond.) 32, 192-200. doi: 10.1038/sj.ijo.0803701

Sharifi, M., Dryden, E. M., Horan, C. M., Price, S., Marshall, R., Hacker, K., etal. (2013). Leveraging text messaging and mobile technology to support pediatric obesity-related behavior change: a qualitative study using parent focus groups and interviews. J. Med. Internet Res. 15:e272. doi: 10.2196/jm ir. 2780

Shaw, K., O'Rourke, P., Del Mar, C., and Kenardy, J. (2005). Psychological interventions for overweight or obesity. Cochrane Database Syst. Rev. 18, CD003818.

Shaw, R. J., Bosworth, H. B., Silva, S. S., Lipkus, I. M., Davis, L. L., Sha, R. S., et al. (2013). Mobile health messages help sustain recent weight loss. Am. J. Med. 126, 1002-1009. doi: 10.1016/j.amjmed.2013.07.001 
Simpson, S. A., Shaw, C., and McNamara, R. (2011). What is the most effective way to maintain weight loss in adults? BMJ 343, d8042. doi: $10.1136 / \mathrm{bmj} . \mathrm{d} 8042$

Simpson, S. G., and Slowey, L. (2011). Video therapy for atypical eating disorder and obesity: a case study. Clin. Pract. Epidemiol. Ment. Health 7, 38-43. doi: 10.2174/1745017901107010038

Swencionis, C., and Rendell, S. L. (2012). The psychology of obesity. Abdom. Imaging 37, 733-737. doi: 10.1007/s00261-012-9863-9

Tufano, J. T., and Karras, B. T. (2005). Mobile eHealth interventions for obesity: a timely opportunity to leverage convergence trends. J. Med. Internet Res. 7:e58. doi: 10.2196/jmir.7.5.e58

Turner-McGrievy, G. M., Beets, M. W., Moore, J. B., Kaczynski, A. T., Barr-Anderson, D. J., and Tate, D. F. (2013). Comparison of traditional versus mobile app self-monitoring of physical activity and dietary intake among overweight adults participating in an mHealth weight loss program. J. Am. Med. Inform. Assoc. 20, 513-518. doi: 10.1136/amiajnl-2012-001510

Villa, V., Manzoni, G. M., Pagnini, F., Castelnuovo, G., Cesa, G. L., and Molinari, E. (2009). Do coping strategies discriminate eating disordered individuals better than eating disorder features? An explorative study on female inpatients with anorexia and bulimia nervosa. J. Clin. Psychol. Med. Settings 16, 297-303. doi: 10.1007/s10880-009-9172-9

Wadden, T. A., Anderson, D. A., Foster, G. D., Bennett, A., Steinberg, C., and Sarwer, D. B. (2000). Obese women's perceptions of their physicians' weight management attitudes and practices. Arch. Fam. Med. 9, 854-860. doi: 10.1001/archfami.9.9.854

Wang, G. J., Volkow, N. D., and Fowler, J. S. (2002). The role of dopamine in motivation for food in humans: implications for obesity. Expert Opin. Ther. Targets 6, 601-609. doi: 10.1517/14728222.6.5.601

Wang, G. J., Volkow, N. D., Logan, J., Pappas, N. R., Wong, C. T., Zhu, W., et al. (2001). Brain dopamine and obesity. Lancet 357, 354-357. doi: 10.1016/S01406736(00)03643-6

Wang, G. J., Volkow, N. D., Thanos, P. K., and Fowler, J. S. (2004). Similarity between obesity and drug addiction as assessed by neurofunctional imaging: a concept review. J. Addict. Dis. 23, 39-53. doi: 10.1300/J069v23n03_04
Wang, G. J., Volkow, N. D., Thanos, P. K., and Fowler, J. S. (2009). Imaging of brain dopamine pathways: implications for understanding obesity. J. Addict. Med, 3, 8-18. doi: 10.1097/ADM.0b013e31819 a86f7

Whitlock, G., Lewington, S., Sherliker, P., Clarke, R., Emberson, J., Halsey, J., et al. (2009). Body-mass index and cause-specific mortality in 900000 adults: collaborative analyses of 57 prospective studies. Lancet 373, 1083-1096. doi: 10.1016/S0140-6736(09)60318-4

Whittaker, R. (2012). Issues in mHealth: findings from key informant interviews. J. Med. Internet Res. 14:e129. doi: 10.2196/jmir.1989

Wilson, G. T. (2011). Treatment of binge eating disorder. Psychiatr. Clin. North Am 34, 773-783. doi: 10.1016/j.psc.2011.08.011

Wing, R. R. (2002). "Behavioral weight control," in Handbook of Obesity Treatment, eds T. A. Wadden and A. J. Stunkard (New York: Guilford Press), 301-316.

Conflict of Interest Statement: The authors declare that the research was conducted in the absence of any commercial or financial relationships that could be construed as a potential conflict of interest.

Received: 04 March 2014; accepted: 20 May 2014; published online: 10 June 2014. Citation: Castelnuovo G, Manzoni GM, Pietrabissa G, Corti S, Giusti EM, Molinari E and Simpson S (2014) Obesity and outpatient rehabilitation using mobile technologies: the potential mHealth approach. Front. Psychol. 5:559. doi: 10.3389/fpsyg.2014.00559 This article was submitted to Psychology for Clinical Settings, a section of the journal Frontiers in Psychology.

Copyright (C) 2014 Castelnuovo, Manzoni, Pietrabissa, Corti, Giusti, Molinari and Simpson. This is an open-access article distributed under the terms of the Creative Commons Attribution License (CC BY). The use, distribution or reproduction in other forums is permitted, provided the original author(s) or licensor are credited and that the original publication in this journal is cited, in accordance with accepted academic practice. No use, distribution or reproduction is permitted which does not comply with these terms. 